\title{
Safety Culture of the Herbert Hoover Dike Contractors
}

\author{
April E. Simons ${ }^{\mathrm{a},{ }^{,},}$Lauren W. Redden ${ }^{\mathrm{a}}$, Matthew Tibbs ${ }^{\mathrm{b}}$ \\ ${ }^{a}$ Auburn University, 270 W. Samford Avenue, Auburn, Alabama 36849, USA \\ ${ }^{b}$ U.S. Army Corps of Engineers, address not available
}

\begin{abstract}
The construction industry remains one of the most dangerous industries to work in even after decades of safety improvements [1]. Researchers, such as Zohar, have argued that safety climate, or safety culture, is "a robust leading indicator or predictor of safety outcomes across industries and countries" [2]. This study made use of mixed methodology that blends quantitative and qualitative research methods to gauge the relative level of commitment each project's workforce has to a positive safety culture at Herbert Hoover Dike. The quantitative portion of the research revealed a positive perception of safety among craft workers. The qualitative portion of the research revealed major themes and subthemes associated with safety culture on the project. Further research is needed to capture additional workers on this project as well as connect safety culture with accident rate with this and other construction projects.
\end{abstract}

(C) 2019 The Authors. Published by Budapest University of Technology and Economics \& Diamond Congress Ltd.

Peer-review under responsibility of the scientific committee of the Creative Construction Conference 2019.

Keywords: Herbert Hoover; safety; safety culture

\section{Introduction}

The construction industry remains one of the most dangerous industries to work in even after decades of safety improvements [1]. While many hazards have been eliminated through legislation and improved safety equipment, unsafe working conditions persists [3]. The safety climate or safety culture of an organization is defined as "product of individual and group values, attitudes, perceptions, competencies, and patterns of behaviour that determine the commitment to, and the style and proficiency of, an organization's health and safety management" [4]. It is the collective values members of an organization share about safety.

Researchers, such as Zohar, have argued that safety climate, or safety culture, is "a robust leading indicator or predictor of safety outcomes across industries and countries" [2]. Published research on worksite safety have argued that it is important to create metrics to measure safety in order to understand whether safety measures actually prevent accidents and illnesses. Measuring performance "1) enables reasoned decisions and assessments, 2) allows comparison with previous (or other's) performance and 3) compares actual performance with planned performances" [5].

The United States Army Corps of Engineers (USACE) relies on lagging indicators to measure safety performance. Lagging, or trailing, indicators are linked to the outcomes of an accident. Lagging indicators include accident reports, OSHA statistics, total recordable index, lost-time index and number of days restricted [6]. Lagging indicators are subject to manipulation by those reporting them. Certain facts may have been omitted, changed, or the incident not reported at all. This research is significant because it provides a snapshot, or baseline, of the safety culture of the contractors performing work at Herbert Hoover Dike (HHD) as measured by leading factors such as confidence in 
management to ensure a safe working environment, self-reported risk taking by the craft workers and work pressure. Existing research suggests that there is no universal metric by which all projects are judged and must be developed for each organization. By creating a baseline through this research, future surveys can be undertaken to measure the positive or negative changes in the safety culture.

\section{Research Summary}

This study made use of mixed methodology that blends quantitative and qualitative research methods to gauge the relative level of commitment each project's workforce has to a positive safety culture at Herbert Hoover Dike.

\subsection{Questionnaires}

Questionnaires were selected as the quantitative method of data collection for this research. The questionnaire was developed in English but a Spanish version was developed as a sizeable percentage of the craft workforce at HHD is native Spanish speakers. Survey takers were asked to answer a series of demographics questions concerning gender, age, length of time in the construction industry, role (prime or subcontractor), and management level (management/supervisor, or worker). Survey takers were also asked to rate a series of twenty-seven questions on a Likert scale from 1 to 5 , with 1 being that they strongly disagreed with the statement and 5 being they strongly agreed with the statement. These Likert scale questions focused on management, safety systems, risk, work pressure and competence. A copy of the complete questionnaire can be found in Appendix A for reference. The candidate population was the entire contractor craft workforce as the research attempted to understand the safety culture of the Herbert Hoover Dike workforce. The survey attempted to reach the greatest breadth of the craft workforce possible. The population of the entire contractor workforce at HHD varies with time but a reasonable estimate is between 50 and 80 individuals on any given day.

Survey data were analyzed using Microsoft Excel. A baseline response was established for each question by averaging all of the survey responses to that particular question. The data was then sorted into subpopulations based on criteria such as age, experience level, and gender. Then, each criteria was compared to the baseline in order to identify any statistically significant differences in the subpopulation mean results by using t-tests (Farrell, 2011).

\subsection{Semi-Structured Interviews}

Semi-structured interviews were chosen as the qualitative method for this research. This structure of interview allows for insights into problems or issues that the questionnaire may not have revealed. The semi-structured interviews were administered to no fewer than six and no more than twelve interviewees as research shows this range is sufficient to draw meaning (Guest et al., 2006). Candidates were selected based on convenience sampling and were selected by the researcher for their close, first-hand knowledge of the site conditions at HHD.

Answers to the interview questions were first entered into Microsoft Word and formatted for ease of reading and coding. Personally identifiable information was then removed to ensure the confidentiality of the interviewee. Important phases and sentences were then given a code for the key idea or theme expressed. The codes were analyzed to develop sub-themes and the sub-themes were then in turn analyzed to produce the principle themes. (Saunders et al., 2009).

\section{Results and Discussion}

\subsection{Questionnaires}

A total of twenty-seven questionnaires were collected. Table 1 shows the frequency of responses from each demographic group and percentage of responses that that group represents out of the total population. 
Simons, Redden, and Tibbs/ Proceedings of the Creative Construction Conference (2019) 099 https://doi.org/10.3311/CCC2019-099

Table 1. Demographics of Questionnaire Respondents.

\begin{tabular}{|c|c|c|c|c|c|c|}
\hline Preferred Language & English & Spanish & & & & \\
\hline & $23(85 \%)$ & $4(15 \%)$ & & & & \\
\hline \multirow[t]{2}{*}{ Gender } & Male & Female & & & & \\
\hline & $24(89 \%)$ & $3(11 \%)$ & & & & \\
\hline \multirow[t]{2}{*}{ Age (years) } & $18-20$ & $21-29$ & $30-39$ & $40-49$ & $50-59$ & $60+$ \\
\hline & $0(0 \%)$ & $1(4 \%)$ & $7(26 \%)$ & $6(22 \%)$ & $9(33 \%)$ & $4(15 \%)$ \\
\hline \multirow[t]{2}{*}{ Experience (years) } & $0-1$ & $2-5$ & $6-10$ & $11-15$ & $16-20$ & $21+$ \\
\hline & $3(12 \%)$ & $4(15 \%)$ & $2(8 \%)$ & $0(0 \%)$ & $5(19 \%)$ & $12(46 \%)$ \\
\hline \multirow[t]{2}{*}{ Employed By } & Prime & Subcontractor & & & & \\
\hline & $17(63 \%)$ & $10(37 \%)$ & & & & \\
\hline \multirow[t]{2}{*}{ Management Level } & Management & Worker & & & & \\
\hline & $14(54 \%)$ & $12(46 \%)$ & & & & \\
\hline
\end{tabular}

As shown in Table 1, the questionnaire respondents were primarily English speaking (85\%), male (89\%), most likely to be 30 years or older and have a mixed of experience levels. There was a skew towards being employed by the prime contractor (63\%) but this is not unexpected as heavy civil work construction often has a high percentage of selfperformed work compare to other construction subsectors. Slightly more than half (54\%) identified themselves as some sort of supervisor or manager.

Figure 1 shows the frequency of responses from each of the individual survey questions (in all categories). The responses to the individual safety culture statements are predominately positive with an average response of a 4.09 (Agree), a standard deviation of 1.17 and a mode of 5 (Strongly Agree). In fact, $78 \%$ of the responses were positive and only $13 \%$ were negative. The remaining $9 \%$ were neutral.

The researcher found no significant difference in the mean responses for the English or Spanish subgroups. The researcher also found no significant difference in the mean responses for the Male or Female subgroups These results suggests that language and gender do not have an impact on the perceptions of the safety culture for those surveyed.

Figure 2 shows the frequency of responses by age range. The researcher found no significant difference in the mean responses for the various ranges of age subgroup. This result suggests that age does not have an impact on the perceptions of the safety culture for those surveyed.

The researcher also found no significant difference in the mean responses for the various ranges of experience of each respondent indicating that years of experience does not have an impact on the perceptions of safety culture for those surveyed. 
Simons, Redden, and Tibbs/ Proceedings of the Creative Construction Conference (2019) 099 https://doi.org/10.3311/CCC2019-099

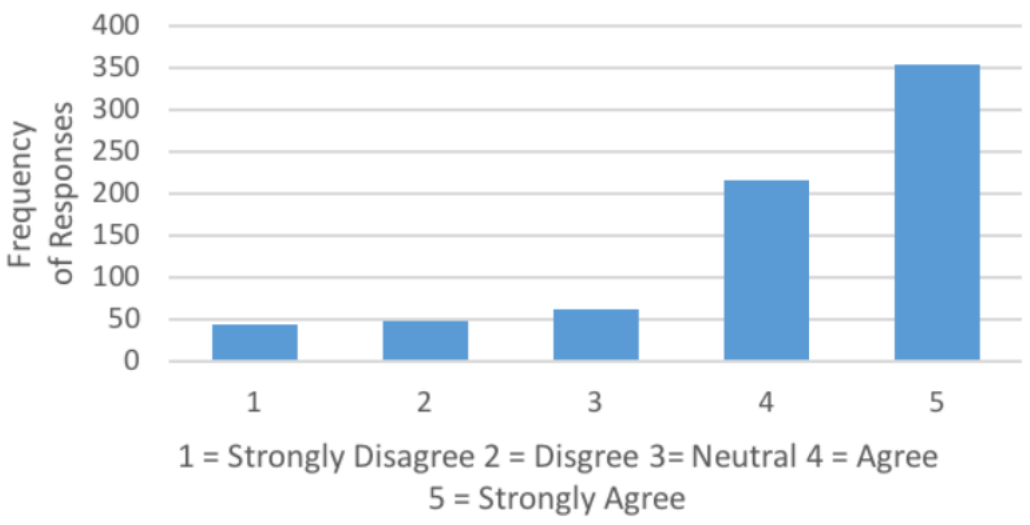

Figure 1: Frequency of Questionnaire Answers

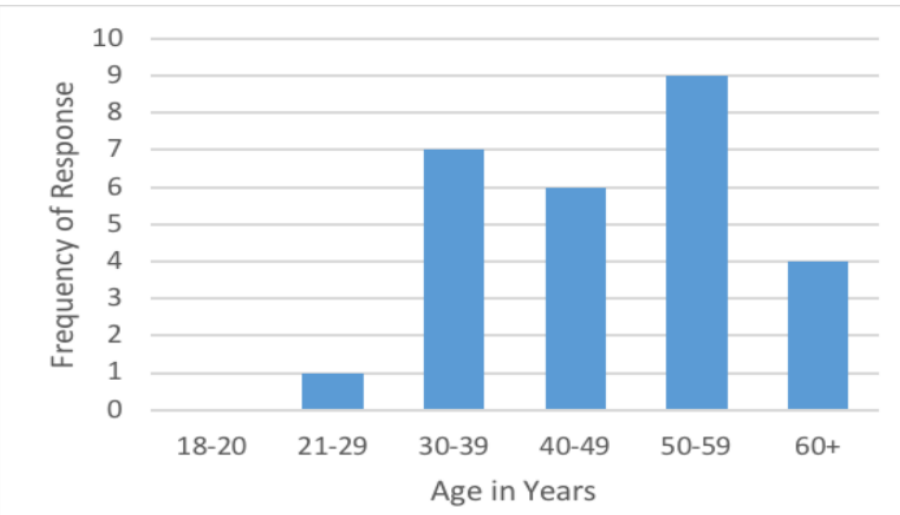

Figure 2: Frequency of Responses by Age

The researcher found no significant difference in the mean responses for the prime contractor or subcontractor subgroups. Nor was there a significant difference between those who identified as management/superintendent or worker. This result suggests that employment with either the prime contractor or subcontractor does not have an impact on the perceptions of the safety culture for those surveyed. The employee's particular role within the company also does not have an impact on perceptions.

\subsection{Semi-Structured Interviews}

Thematic analysis was performed on the interviews to identify the themes common between the interviewees. The analysis uncovered the following three major themes: (1) Management, (2) Worker and (3) Safety Officer. Each major theme had associated subthemes which are shown in Figure 3.

The management theme includes all of the items that the site management team has control over, the motivations for maintaining site safety, and the situations in which the owner must intervene. 
Simons, Redden, and Tibbs/ Proceedings of the Creative Construction Conference (2019) 099 https://doi.org/10.3311/CCC2019-099

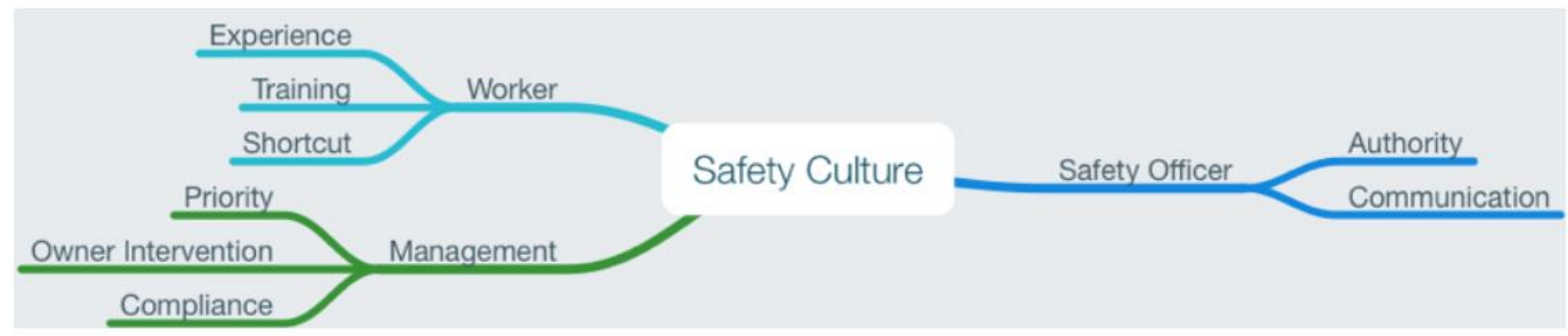

Figure 3: Safety Culture Interview Thematic Analysis

The priority subtheme encompasses the apparent level of concern, or priority, the site management places on safety. One interviewee stated, "the site management I've been involved with has a positive attitude regarding safety" and that they "seem fairly proactive." The other interviewees noted a similar general positive attitude towards safety. It was also noted in several of the interviews that management seems to prioritize safety hazards by severity. Interviewee 3 stated that "some [safety] deficiencies take longer" but "deficiencies that can pose immediate danger to the employees are addressed almost immediately"

Owner intervention was another subtheme found within the interviews. This subtheme includes the necessity of owner intervention in the site management's safety efforts. The level of intervention described in the interviews varies from the occasional reminder to demands for the site management to terminate employees. Overall, the theme was that owner intervention is required at some level.

The Compliance subtheme includes the motivations of why the site management maintains an adequate safety program. The conclusion drawn from the statement made by the interviewees is that safety is largely a contractual and legal compliance issue. One interviewee said that site management corrects safety deficiencies because the deficiencies "threaten [s] to impact work performance"

The worker theme includes ways in which the worker's attitudes, behaviors and knowledge has an impact on his or her own personal safety.

The experience subtheme encompasses the role in which an individual worker's experience plays in the choices he or she makes on the jobsite. One interviewee discussed how more experienced workers are more safety conscious in this quote: "older guys who have been around have seen [accidents] happen and know that all it takes is someone not paying attention and they can get hurt." In a different interview the following quote discusses how inexperienced workers are less safety conscious: "labor for hire workers...seem to be bothered by some of the basic safety equipment, like properly wearing PPE."

Training is a common subtheme in the interviews in how it pertains it is important in imparting the skills necessary to the workers to identify hazards on the jobsite. This can be seen in the follow quote: "Many employees can identify safety hazards but to accomplish this the contractor need to have a good safety program that keeps the employees aware of the risks."

Shortcut taking was identified by the researcher as another subtheme. This subtheme includes the propensity of otherwise experienced, training, and safety conscious workers to take risks on the jobsite. The interviewees believed most shortcut taking was done out of "laziness" or a worker being "in a hurry" or overconfidence. Shortcut taking was also noted to be done when a worker's supervisor or the site safety officer was not present.

The safety officer theme includes the role the safety offers plays in administering the safety program and the authority vested in him or her. The safety officer, while not always explicitly named, is a central figured discussed in the interview.

Authority of the safety officer is a subtheme that involves the authority and responsibility delegated to the safety officer on site. The interviewees noted that the safety officer "have the authority to stop work if there is a safety risk." One interviewee stated that a crane operator was "suspended for 3 days from the site" for "disobeying the SO order." This is evidence of site managements inherit support of the authority of a safety officer. 
Simons, Redden, and Tibbs/ Proceedings of the Creative Construction Conference (2019) 099

https://doi.org/10.3311/CCC2019-099

The communication subtheme outlines how training and information is imparted to the worker through the safety officer. This is accomplished through weekly toolbox meetings, new worker safety indoctrinations and activity hazard analyses.

\section{Conclusions and Recommendations}

For survey data gathered from craft workers, the responses to the individual safety culture statements were predominately positive with an average response of a 4.09 (Agree), a standard deviation of 1.17 and a mode of 5 (Strongly Agree). The researcher found no significant difference in the mean responses for the following subgroups:

- English or Spanish Speaking

- Male or Female

- Age Range

- Years of Experience

- Employed by Prime Contractor or Subcontractor

- Holding a Management/Superintendent or Worker Position

The results indicate that none of the factors listed above have an impact on perceptions of safety culture for those surveyed.

The semi-structured interviews revealed three three major themes: (1) Management, (2) Worker and (3) Safety

Officer. Under management, the priority subtheme revealed a positive attitude toward safety from site management. Owner intervention was identified as management subtheme, indicating the perception among interviewees that owner intervention is required for success. For the compliance subtheme, the interviewees revealed the perception that safety is largely a contractual and legal compliance issue.

Under the worker theme, the experience subtheme encompasses the role in which an individual worker's experience plays in the choices he or she makes on the jobsite. The perception was generally that more experience leads to more safety consciousness. Worker training was also identified as an important subtheme in terms of transferring skills necessary to identify jobsite hazards. Interviewees also identified shortcut taking as a worker subtheme, citing many workers taking shortcuts when in a hurry, overconfident, or when the safety officer is not present.

The safety office theme revealed subthemes of authority and communication. Interviewees indicated that safety officers have authority and responsibility to stop work that is unsafe. Communication refers to the transfer of training from the safety officer to workers through meetings and training.

Further research should aim to administer the questionnaire again in six months to a year and analyze the changes, if any, in the data. The sampling on a particular site should also occur over several days to capture the more transitory, and the potential more venerable, craft workers. Future research could also be undertaking to establish a more causal relationship between safety culture and accident rates on the job site. This is currently not well explored in the available literature.

\section{Appendix A. Questionnaire in English}

\section{Demographics}

1. Are you male or Female

Male

Female 
Simons, Redden, and Tibbs/ Proceedings of the Creative Construction Conference (2019) 099 https://doi.org/10.3311/CCC2019-099

2. What is your age?

$18-20$

21-29

30-39

40-49

$50-59$

60 or older

3. How long have you worked in the construction industry?

$0-1$ years

$2-5$ years

6-10 years

11 - 15 years

$16-20$ years

21 or more years

4. Do you work for the Prime Contractor or a Subcontractor?

Prime Contractor

Subcontractor

5. Are you a management/supervisor or are you a worker?

Management/Supervisor or Worker

Likert-type scale: (strongly disagree, disagree, neutral, agree, strongly agree)

\section{Management}

6. I feel that safety is as important as production to management.

7. Management listens to me when I raise safety concerns.

8. Safety hazards are quickly fixed by management.

9. Management only responds to safety hazards when there are accidents.

10. Employees that work safety are praised by management.

11. Safety lessons learned are shared with everyone on the site.

Safety Systems

12. The rules are there to keep me safe. 
Simons, Redden, and Tibbs/ Proceedings of the Creative Construction Conference (2019) 099

https://doi.org/10.3311/CCC2019-099

13. Safety rules are clearly explained.

14. Safety rules get in the way to doing the work.

15. The safety procedures are too complicated for me to follow.

16. Safety personnel support me working safety.

17. The housekeeping on the site is a priority.

18. I am provided the safety equipment I need for the hazards I face on the jobsite.

Risk

19. Safety equipment is only necessary for novices.

20. I work areas I know there are safety hazards.

21. I find PPE, such as hard hats, gloves, and fall protection, uncomfortable.

22. When my supervisor isn't around I don't follow the proper procedures.

23. I continue to use equipment that have broken or disabled safety features.

24. I don't think that I will be involved in an accident this year.

\section{Work Pressure}

25. I work under a great deal of pressure to get the job done.

26. Safety can get in the way of production.

27. I take shortcuts in safety to meet production goals.

28. Coworkers take shortcuts in safety in order to meet production goals.

\section{Competence}

29. I receive the training I need to work safe.

30. I don't know how to use the safety equipment I need to work safe.

31. I am able to identify safety hazards in my work area.

32. I actively look for safety hazards on the jobsite.

\section{References}

[1] Holden, M., 2016. 2015-2016 Construction Safety Highlights - [WWW Document]. Occup. Health Saf. URL https://ohsonline.com/articles/2016/09/01/2015-2016-construction-safety-highlights.aspx (accessed 9.19.16).

[2] Zohar, D., 2010. Thirty years of safety climate research: Reflections and future directions. Accid. Anal. Prev., Safety Climate: New Developments in Conceptualization, Theory, and Research 42, 1517-1522. doi:10.1016/j.aap.2009.12.019

[3] Langford, D., Rowlinson, S., Sawacha, E., 2000. Safety behaviour and safety management: its influence on the attitudes of workers in the UK construction industry. Eng. Constr. Archit. Manag. 7, 133-140. doi:10.1046/j.1365-232x.2000.00137.

[4] Health and Safety Executive, 1993. ACSNI Study Group on Human Factors: Third Report - Organizing for Safety. HSC (Health and Safety Commission), London, United Kingdom.

[5] Ingalls, T.S., 1999. Using scorecards to measure safety performance. Prof. Saf. 44, 23-28.

[6] Toellner, J., 2001. Improving safety \& health performance: Identifying \& measuring leading indicators. Prof. Saf. 46, $42-47$. 